\title{
Error Value Detection and Correction for Detumbling using Magnetometer, GPS and Kalman Filter for STUDSAT - 2
}

\author{
H. Venkatesh Kumar \\ Professor, \\ Electronics and Communication \\ Engineering, \\ Nagarjuna College of Engineering \\ \& Technology, Bengaluru, India
}

\author{
Indukuri Goutham Sanjay \\ Varma \\ Student, \\ Electronics and Communication \\ Engineering \\ Nagarjuna College of Engineering \\ \& Technology, Bangalore, India
}

\author{
P. Maharshi Naveen \\ Student, \\ Electronics and Communication \\ Engineering, \\ Nagarjuna College of Engineering \\ \& Technology, Bengaluru, India
}

\author{
Obinaboina Vengaiah \\ Student, \\ Electronics and Communication Engineering \\ Nagarjuna College of Engineering \& Technology \\ Bengaluru, India
}

\author{
Mahitha R. \\ Student, \\ Electronics and Communication Engineering \\ Nagarjuna College of Engineering \& Technology \\ Bengaluru, India
}

\begin{abstract}
STUDSAT-2 is a STUDent SATellite program that aims to build Twin Nanosatellites each weighing approximately $10 \mathrm{~kg}$ to demonstrate InterSatellite Communication to improve temporal resolution for remote sensing applications. This paper focuses on attitude determination (ADCS) for small satellites. The method described uses a magnetometer to resolve the three-axis attitude of the satellite. The primary challenge is that magnetometers only instantaneously resolve two axes of a satellite's attitude. Typically, magnetometers are used in conjunction with other sensors to resolve all three axes. In our project, we are using the STM32F407G discovery board controller with a magnetometer and GPS to monitor the change in the attitude of the satellite, and actuator coils to reduce the tumbling rate. By using the IGRF/ECER, model as a reference for the standard magnetometer values. The principle of the Kalman Filter algorithm is used to predict the position of the satellite by taking the inputs from the magnetometer and GPS.
\end{abstract}

\section{Keywords}

STUDSAT-2, Attitude Determination and Control system (ADCS), Kalman Filter, Sensors.

\section{INTRODUCTION}

STUDSAT is a student satellite program continuing the legacy of STUDSAT-1, India's first picosatellite, undergraduate students from a consortium of seven engineering colleges across Karnataka have undertaken Project STUDSAT- 2. It is India's first student twin NanoSatellite mission which aims to demonstrate InterSatellite communication between two Nano-satellites STUDSAT2A/2B. The mission objectives of Project STUDSAT-2 are to demonstrate In-orbit satellite separation, Inter-satellite communication between STUDSAT-2A and STUDSAT-2B, the Implementation of Drag Sail Technology in (STUDSAT2B) for de-orbiting and capturing images of Earth with a CMOS Multispectral camera.

Among the sensors, STUDSAT-2 employs sun sensors, magnetometer, and gyro sensors to sense the attitude of the satellite. Attitude Determination and Control System (ADCS) is responsible for maintaining the proper orientation of the satellite during a specified orbit period. The appropriate sensors and actuators have been studied and chosen considering various aspects including the mission requirement, power constraints, and cost.

\section{RELATED WORK}

First, In the paper "A high precision attitude determination and control system for the UYS-1 nanosatellite" by Jefferson R. Chaurais, Henrique C. Ferreira, João Y. Ishihara, Renato A. Borges, Anatoliy M. Kulabukhov, Vladimir A. Larin, Vladimir V. Belikov [1], the design of a high precision attitude determination and control system for the UYS-1 Ukrainian nanosatellite. Its main task is the 3-axis stabilization with less than 0.5-degree angle errors, so the satellite may take high precision photos of Earth's surface. To accomplish this task, this system comprises a star tracker and three reaction wheels. To avoid external disturbances and actuators faults, a PD-type and PID-type robust controllers are simulated and the results are compared to an empirically adjusted PD controller.

In the paper "Performance analysis of micrium RTOS in the computer of a nano-satellite" by Chandrasekhar Nagarajan, Krishna Kinger, Faraz Haider, Rajat Agarwal [2], data handling and power management is a vital aspect to mission life. The computer acts as the brain of a satellite, which is expected to operate at constrained resource environments. A real-time operating system is used in such cases where deterministic execution is of prime importance. This paper describes how the real-time operating system is ported to an STM32F207 to control the satellite's mission life. A comparative study of how this RTOS is best suited for our mission is also presented. The RTOS is specifically designed to effectively manage power in this dual controller system. Results of 14-orbit simulation for control and attitude determination algorithms are discussed. The software design here is expected to monitor and control the execution of both the onboard controllers. The design of the software will be compatible with other university missions as well because most of them base their design on a similar hardware bus. 
In the paper "Development testing and selection of attitude determination algorithm for STUDSAT-2" by Divyanshu Sahay, Navaneet Villodi, S Sandya [3], describes the development, testing, and selection of an attitude determination algorithm for STUDSAT2 (STUDent SATellite). The algorithms, namely TRIAD, Optimized TRIAD, Davenport's Q and QUEST are considered. For testing, these are coded in MATLAB and then compared based on errors in the angle of rotation about the Gibbs vector. The least-squares criterion is accounted for, by minimization of the loss function. Computational efficiency is accessed using stand-alone precompiled optimized codes in embedded $\mathrm{C}$ language.

In the paper "Design and Development of 3-axis Reaction Wheel for STUDSAT-2" by Kumar Saroj, Divyanshu Sahay, Sandesh R. Hegde, S. Sandya, Anik Kumar Jha, T. C. Mahalingesh [4], describes the designing, detailed motion, and frequency analysis and brief control approach of nano reaction wheels that have to be used as an actuator for STUDSAT-2. The designing part comprises of designing a flywheel, tetrahedral configuration explanation, and the motor selection. The worst-case disturbance modeling is done to design the flywheel considering the four primary environmental disturbances namely aerodynamic drag, solar radiation, magnetic residual and gravity gradient. Using the design parameters a study is performed to minimize the mass of the flywheel.

In the paper "Research on the power management system of manned spacecraft" by R. Xiaoqiang, L. Xiaofeng, and Y. Liman [5], the use of small satellite technology has grown to such lengths that they have proven to be a viable solution for space science and exploration, rivaling traditional satellites. They are composed of a variety of subsystems: communication, on-board computer, attitude determination, and control as well as an electronic power supply. Power is provided by solar panels mounted on each one of the CubeSat sides. Storage is used to supply power when the CubeSat solar panels are shaded by Earth's shadow region, where sunlight is not readily available. Surface area limitations create a challenge for most CubeSats, reducing solar panel generation.

\section{METHODOLOGY}

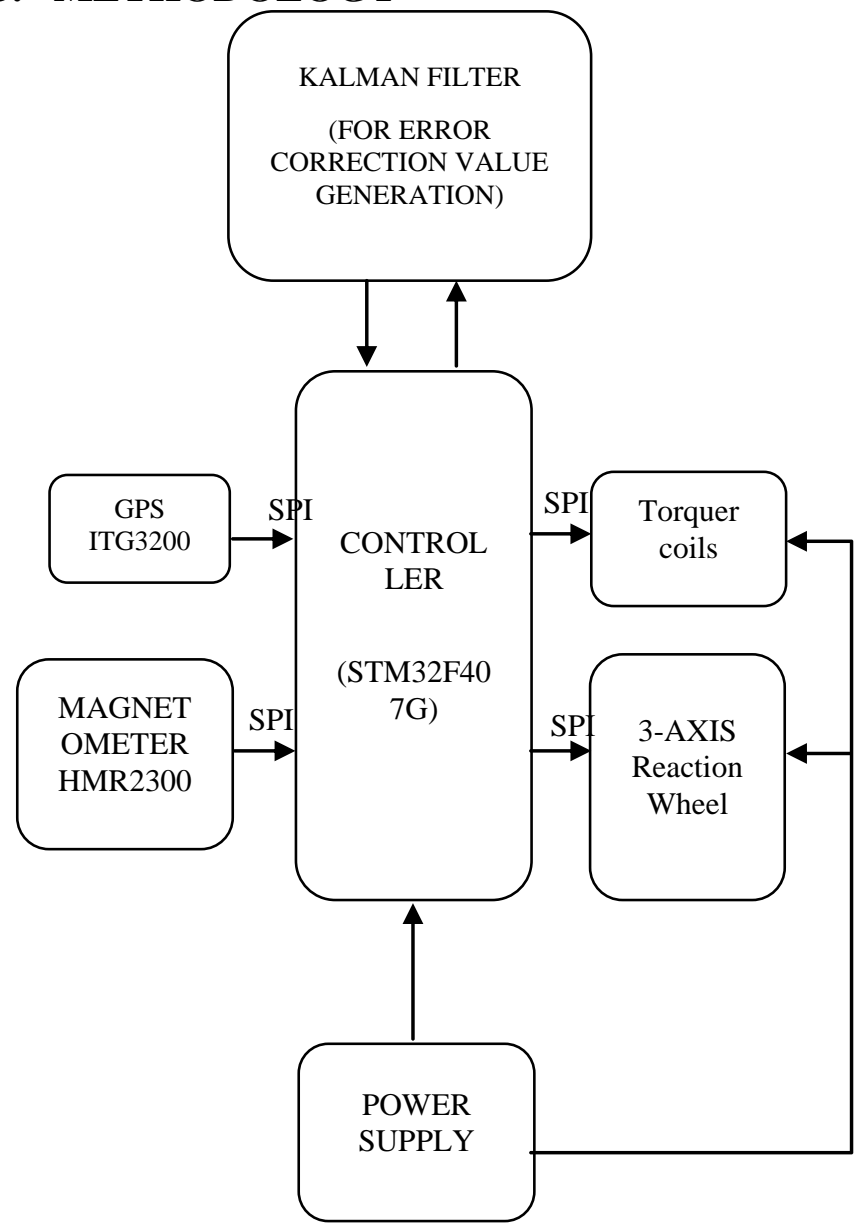

Fig 3.1: System Architecture

The STM32F407G microcontroller and sensor modules are connected by SPI. The magnetometer sensor senses the three dimensional magnetic values of the satellite. During tumbling the magnetic values of the satellite, continuously changes and which are given by the magnetometer, which is compared with the IGRF model that gives the standard magnetic values and the difference of the error is noted. Kalman filter algorithm which is used as a predictor. The algorithm takes the inputs from GPS and predicts the further location of the satellite. In the predicted GPS locations the standard magnetic values are noted with help of the IGRF model and the actual magnetic values are noted with a magnetometer and based on the difference between them torquer coils are rotated such a way that the satellite is facing towards the earth.

After Ejecting the satellite into the space in its orbit, due to the ejection force the satellite tends to tumble and there will be huge fluctuation in attitude of the satellite. With the uncertainty in attitude the satellite cannot transmit data, for that attitude determination and detumbling is required. The information is gathered from the initialized magnetometer sensor and global positioning system. The micro controller is programmed to detect the tumbling and if the satellite is tumbling, for getting the required RPM to detumble pass the GPS and magnetometer values to the Kalman filter and get the real time detumbling rate values. 


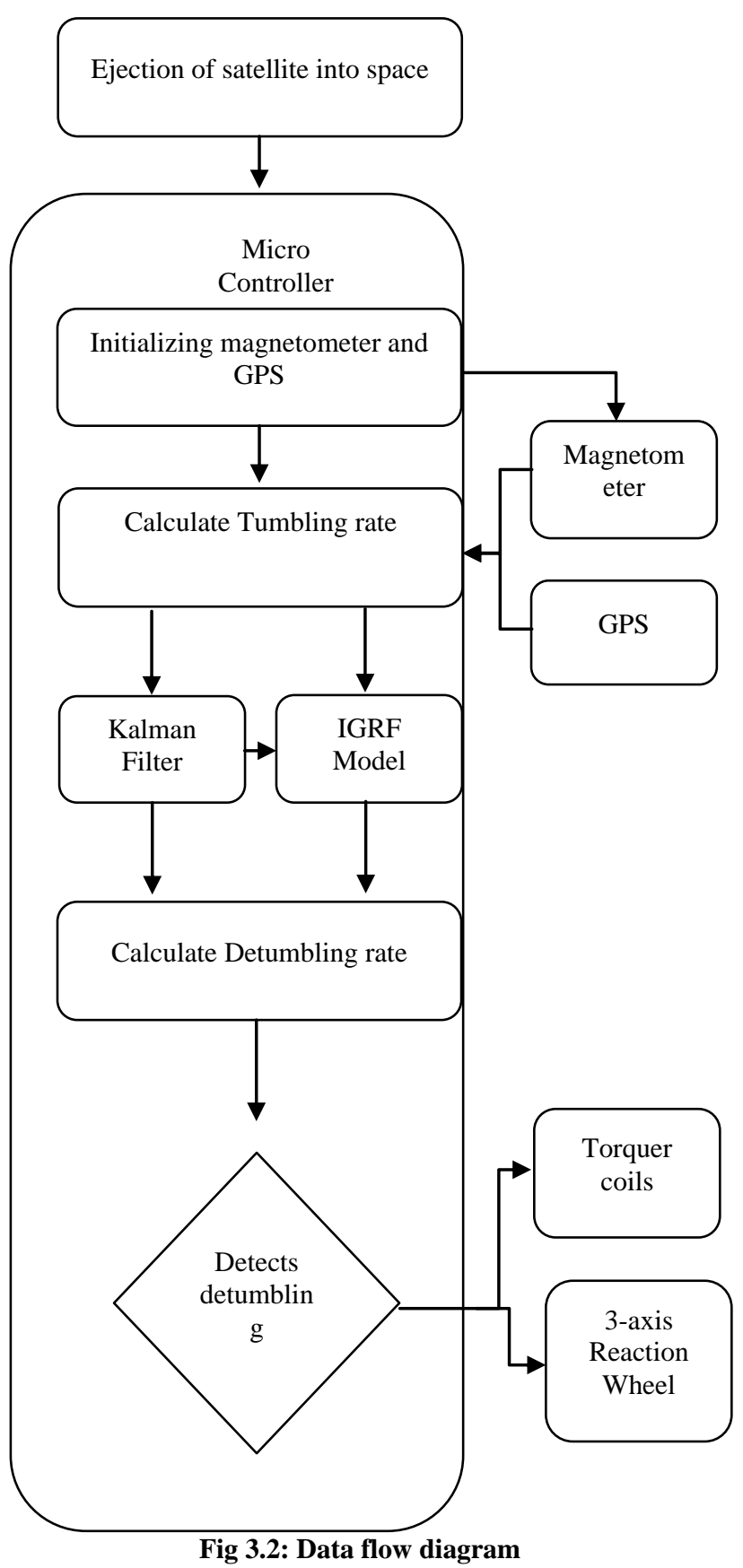

These values are fed to actuators for correcting the Location.

\section{WORKING}

The attitude of a spacecraft is its orientation in space The Attitude Determination and Control System (ADCS) is a crucial subsystem of a spacecraft. It provides pointing accuracy and stability of the payloads and antennas as critical parts of the system and control operation and the mission success. During imaging, the satellite must point towards the earth to get precise readings, which is accomplished by the ADCS. In this work a magnetometer, GPS and Extended Kalman filter are used. When the satellite is launched, it undergoes tumbling to DE tumble the satellite the above-mentioned sensors are used.

During tumbling the magnetic values of the satellite, continuously changes and which are given by the magnetometer, which is compared with the IGRF model that gives the standard magnetic values and the difference of the error is noted. Extended Kalman filter (EKF) algorithm which is used as a predictor, Using the EKF for predicting the GPS locations of the satellite by taking the inputs from the GPS sensor. In the predicted GPS locations the standard magnetic values are marked with help of the IGRF model and the actual magnetic values are monitored with a magnetometer and based on the difference between them torquer coils are rotated such a way that the satellite is facing towards the earth.

\section{RESULTS DISCUSSION}

According to Fig 3.3 whenever the satellite is accelerated towards $\mathrm{X}$-axis, then the pitch of the satellite will be varying and it needs number of acceleration shots to get the pitch stable. As the antenna should face the earth the pitch of the satellite should be appropriate.

According to Fig 3.4 whenever the satellite is accelerated towards Y-axis, then the Yaw of the satellite will be varying and it needs number of acceleration shots to get the Yaw stable. As the payload should be in appropriate position, we need to stabilize yaw of the satellite.

According to Fig 3.5 whenever the satellite is accelerated towards Z-axis, then the satellite will Roll and there will be change in both the axis and it needs number of acceleration shots in both the directions to get the Roll stable. As both the antenna and payload should be in appropriate position, we need to stabilize Roll of the satellite.

As shown in Fig 3.6 the satellite works efficiently if it is in the proper axis. The satellite should detumble and aligned in the prefixed axis for getting the efficient results. 
VarViewer1

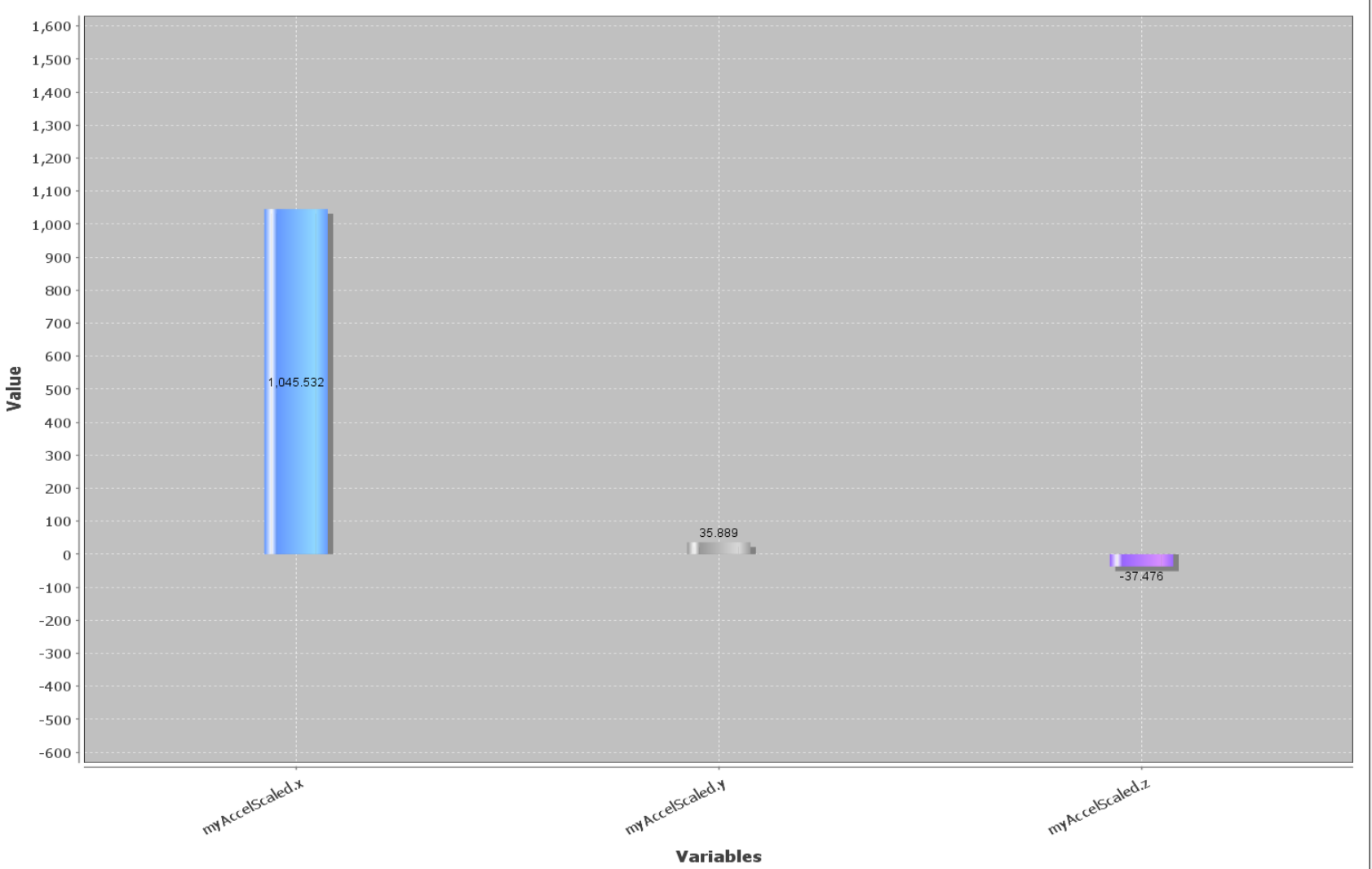

Fig 3.3: Acceleration towards $X$-axis

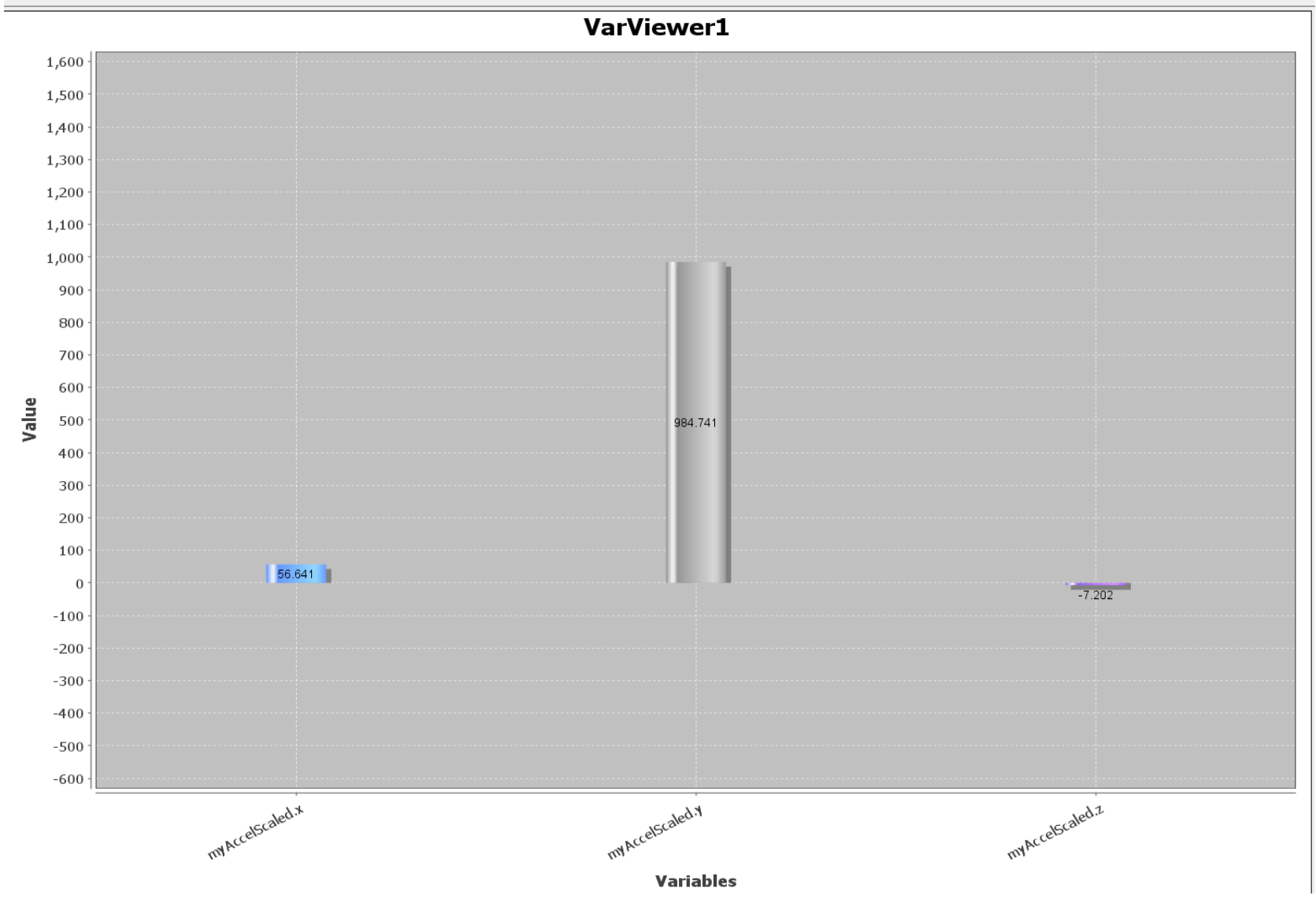

Fig 3.4: Acceleration towards $Y$-axis 
VarViewerl

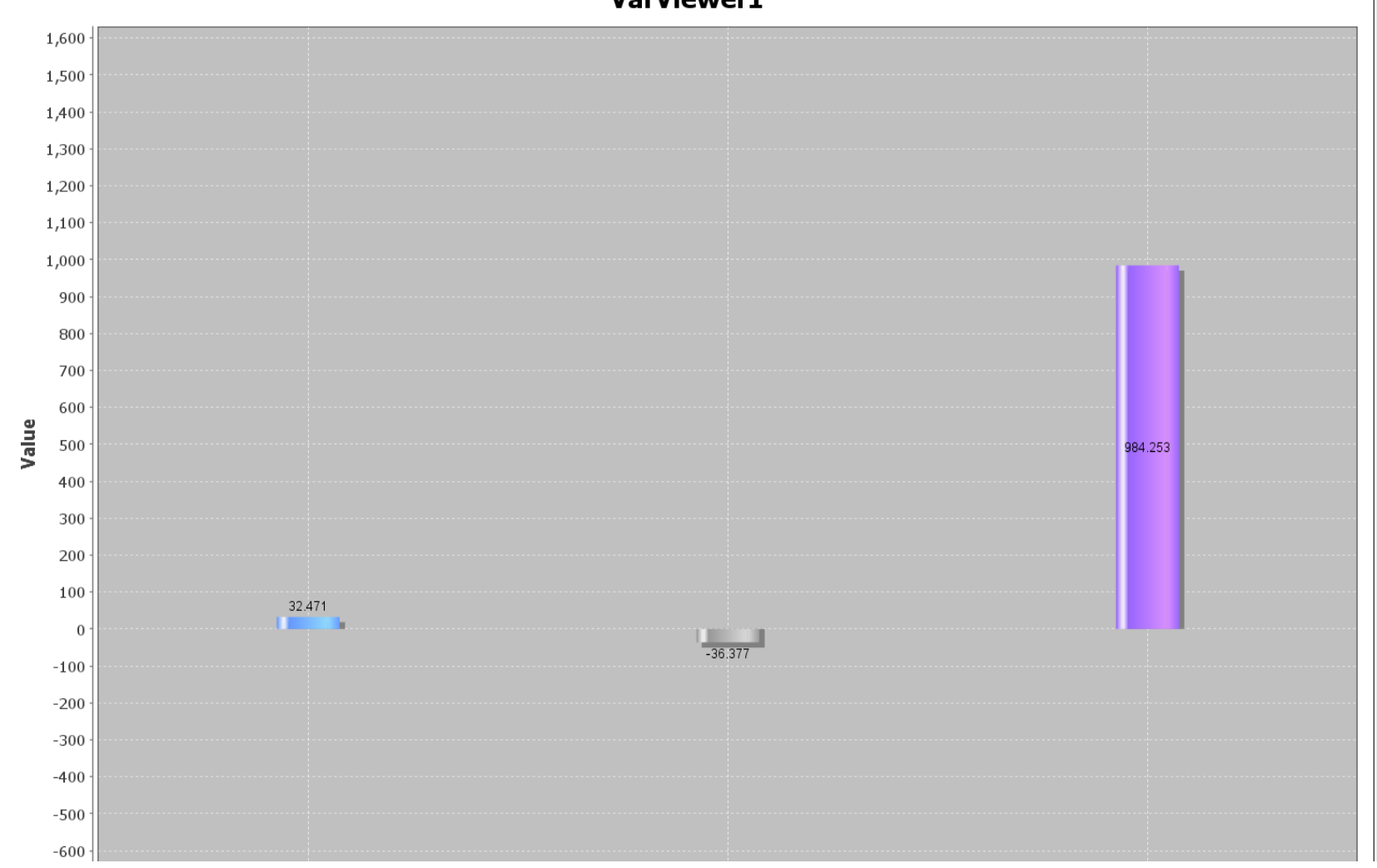

Fig 3.5: Acceleration towards Z-axis

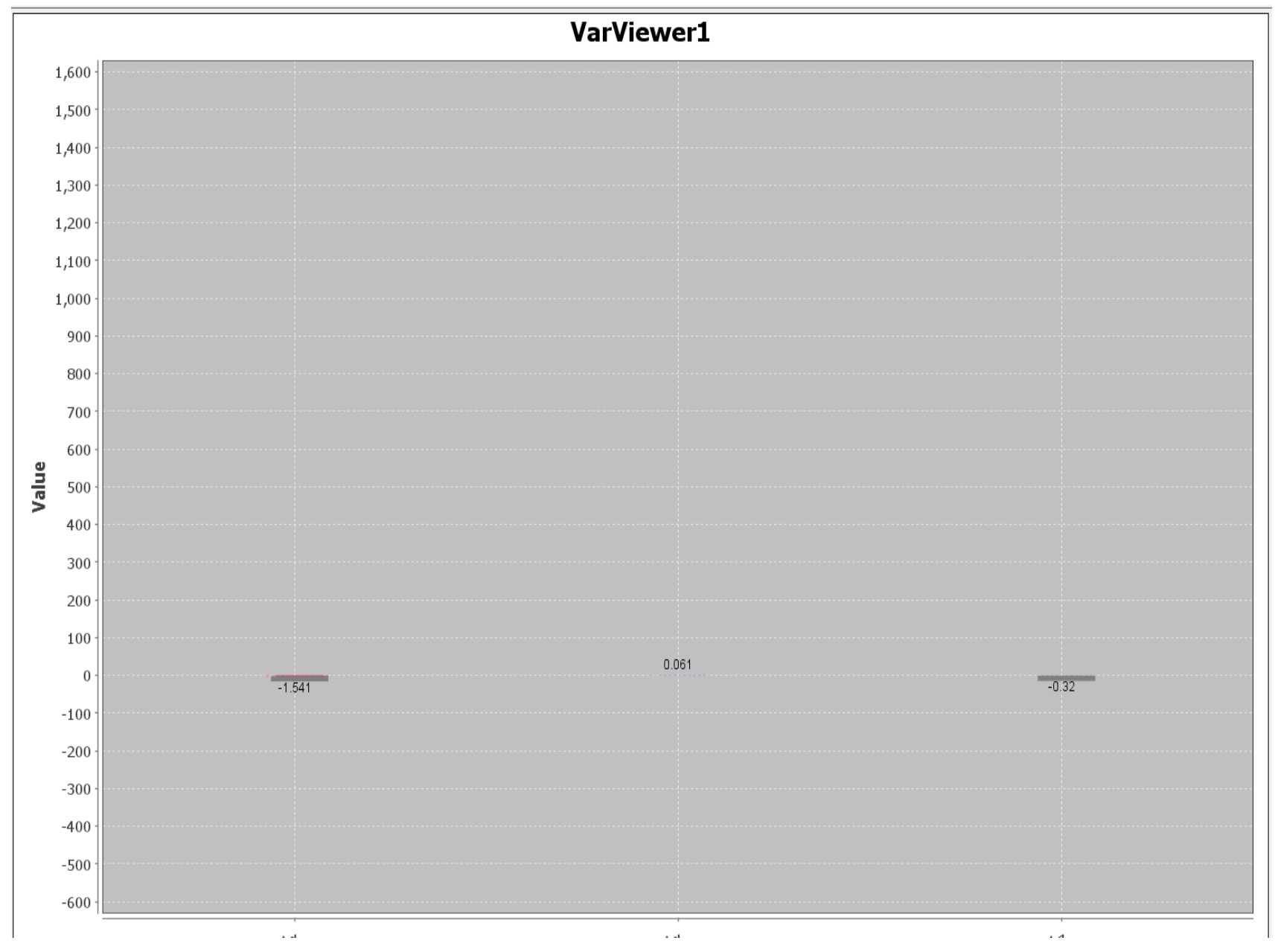

Fig 3.6: Acceleration is zero in all axis's 


\section{CONCLUSION}

A few conventional control methods adopted for the microsatellite for spin stabilization have been simulated and results are highlighted in this work. This proves the stability by Kalman filter using the values of magnetometer and GPS location. A Kalman Filter algorithm that takes inputs from the magnetometers and GPS sensors has been developed and implemented in realistic simulations, using this we can find the pointing accuracy and spin rate error. With the estimator in the loop, the system worked best with the energy controller where there was an accuracy loss that is obtained. The obtained results proves that these outperform the benchmark of a combination of the magnetometer, GPS Sensing modules regarding the accuracy of attitude determination.

\section{ACKNOWLEDGEMENT}

We would like to express our gratitude to STUDSAT-2 research center, NMIT for providing facilities to carry out this project and also we express our sincere thanks to the research associates of NMIT, Bangalore and internal STUDSAT-2 coordinator Dr H Venkatesh Kumar for extended support.

\section{REFERENCES}

[1] Jefferson R. Chaurais, Henrique C. Ferreira, João Y.
Ishihara, Renato A. Borges, Anatoliy M. Kulabukhov, Vladimir A. Larin, Vladimir V. Belikov, "A high precision attitude determination and control system for the UYS-1 nanosatellite", Aerospace Conference 2013 IEEE, pp. 1-12, 2013.

[2] Chandrasekhar Nagarajan, Krishna Kinger, Faraz Haider, Rajat Agarwal, "Performance analysis of micrium RTOS in the computer of a nano-satellite", Aerospace Conference 2015 IEEE, pp. 1-9, 2015.

[3] Divyanshu Sahay, Navaneet Villodi, S Sandya, "Development testingand selection of attitude determination algorithm for STUDSAT-2", Aerospace Conference 2017 IEEE, pp. 1-8, 2017.

[4] Kumar Saroj, Divyanshu Sahay, Sandesh R. Hegde, S. Sandya, Anik Kumar Jha, T. C. Mahalingesh, "Design and development of 3-axis reaction wheel for STUDSAT-2", 2015 IEEE Aerospace Conference, 2015.

[5] R. Xiaoqiang, L. Xiaofeng and Y. Liman, "Research on power management system of manned spacecraft," 6th IEEE Conf. on Industrial Electronics and Applications, 2011, pp. 19281932. 